\title{
Safety and Feasability of Muslim Fasting While Receiving Chemotherapy
}

\author{
Talha Badar $^{1}$, Abdelsalam Ismail ${ }^{2,3}$ \& Ali AlShanqeeti ${ }^{1}$ \\ Heamatology 1 and Medical Oncology Department2 , Comprehensive Cancer Centre, King Fahad Medical City, \\ P.O. Box 59046. Riyadh 11525, Kingdom of Saudi Arabia. \\ 3 Clinical Oncology Department, Faculty of Medicine, Alexandria University. \\ Alexandria, Egypt.
}

\begin{abstract}
More than 1.2 billion Muslim practice fasting from dawn to sun set through the whole month of Ramadan. Cancer patients who are on active chemotherapy therapy treatment are usually feeling impressed whether or not to keep fasting with no data to support the possible hazard or potential benefit, Here we report data about representative samples from our patients who had been followed during the fasting time and use them as referral control for themselves while receiving the chemotherapy without fasting. Subjective symptoms and objective laboratory results are reported. To our knowledge this is the first ever study in the field.
\end{abstract}

\section{INTRODUCTION}

Throughout the holy month of Ramadan, Muslims fast from dawn to sunset by refraining from all forms of eating and drinking. Fasting is exempted for, among others, those who are sick. Oncologists practicing in Muslim countries are always asked by their patients whether they can observe fasting while receiving their chemotherapy during Ramadan. Moreover, it has also been our anectodotal observation that some patients insist on observing their fasting during the month of Ramadan, irrespective of their physician advice. There is currently no consensus among oncologists regarding the recommendation to fast or not fast among these patients. To our knowledge, this issue has not been addressed in a previous study or a clinical trial.

Diet has strong relation with occurrence of cancer and has been estimated by the American Institute for Cancer Research and the World Cancer Research Fund that 30-40 percent of all cancers can be prevented by healthy lifestyle measures. In animal models, calorie restriction reduces incidence of spontaneous cancers and protects against carcinogen-induced cancers. ${ }^{2}$ With calorie restriction, tumor incidence, multiplicity, and burden are all reduced significantly. ${ }^{3,4}$ Calorie restriction has been shown in rats to be effective even in the face of high fat diets. ${ }^{4}$ Ross and Bras ${ }^{5}$ demonstrated that when the energy intake of rats was restricted for only the first 7 weeks of life, the incidence of spontaneous tumors fell by $40 \%$ while Weidruch and Walford showed that calorie restriction is effective even when instituted late in mice life ${ }^{6}$. In addition, starvation gives normal cells an advantage over cancer cells in resisting oxidative stress when exposed to chemotherapeutic agents. In a recent study, Raffaghello et al, ${ }^{7}$ showed that fasting prior to cancer chemotherapy treatment may significantly enhance the cancer-killing effects of the drugs while protecting healthy cells from damage in mice. All mice were given an amount of the cancer drug etoposide equivalent to three times the maximum human dose. In their experiments Raffaghello et al demonstrated that low glucose or low-serum media (24-48 hours) protected primary glial cells but not six different rat and human glioma and neuroblastoma cancer cell lines against hydrogen peroxide or the chemotherapy drug cyclophosphamide. Furthermore, short-term starvation (48 hours) provided complete protection to mice but not to injected neuroblastoma cells against a high dose of the chemotherapy drug etoposide. Lee et $\mathrm{al}^{8}$ and Raffaghello et $\mathrm{al}^{7}$ found out in their studies that fasting causes reduction of circulating insulin like growth factor 1(IGF-I) which protects normal mice cells against doxorubicin, cyclophosphamide and 5-fluorouracil (5-FU) cytotoxicity.

In humans alternate day calorie restriction has shown to improve symptoms and clinical finding in obese asthmatic patients and was well tolerated. ${ }^{9}$ Even in children from 6 months to 15 years 14-40 hrs of complete fasting was well tolerated in a clinical study done in Philadelphia, USA. ${ }^{10}$ These studies suggest that caloric restriction has been shown not only that it is well tolerated by humans but also reduces markers of inflammation and oxidative stress. ${ }^{9,11}$ Safdie et al ${ }^{12}$ reports 10 cases diagnosed with variety of cancers who voluntary fasted All for a total of 48 to 140 hours prior to and/or 5 to 56 hours following chemotherapy. Results from this study suggested that chemotherapy while fasting is feasible and safe. Moreover, fasting while chemotherapy has a potential to reduce side effects related to chemotherapy.The dietary recommendation for cancer patients receiving chemotherapy, as described by the American Cancer Society, is to increase calorie and protein 
intake. ${ }^{13}$ However in simple organisms, mice, and humans, dietary restriction induces wide-ranging changes associated with cellular protection and limit the chemotherapy associated cellular toxicity. ${ }^{6-8,12,14}$ However we are lacking data that apply to Muslims fasting which is rhythmic from sunrise to sunset, devoid of food and drinks completely pertaining to that period and being practiced by more than 1.8 billion Muslims across the globe every year for period of one month.

\section{OBJECTIVE}

We propose to conduct a pilot study during the Islamic months of Ramadan and Shawwal on patients receiving chemotherapy for various cancers. The aim of the study is to assess feasibility and safety of fasting before and after receiving chemotherapy. In addition, this study will compare the impact of fasting versus nonfasting on the side effect profile of the chemotherapeutic regimen received.

\section{METHODS}

\section{Design and study overview}

This was a one-group non-randomized, cross-over, , open-label, safety and feasibility pilot study.Eleven patients were recruited: fasting, patients receive their previously chemotherapy 20 minutes after sunset, and then allowed to continue their routine fasting schedule for the rest of the month (i.e., fast daily from dawn to sunset and eat and drink from sunset to dawn). After a "wash out" period of at least two weeks after end of Ramadan, patients receive the same chemotherapy while not fasting. All patients interviewed by phone daily regarding the presence or absence of chemotherapy side effects, and a complete blood count (CBC) as well as renal and liver function (RF and LFT) monitored once weekly. All laboratory tests were done 10 am for all patients as well as immediately before starting chemotherapy during the fasting period.

\section{Study duration}

The study was done in the second half of Ramadan, after 15/9/1429 (15/9/2008), and continue till the end of Shawwal (October 2008) with at least 2 weeks "washout period" after the end of fasting. Patients were active in the study for 4 weeks, 2 during Ramadan and 2 during Shawwal, starting no earlier than the second week in each month.

\section{Endpoints}

Primary endpoint: Safety of fasting while receiving chemotherapy. This was evaluated by using the blood biomarkers of White Blood Cell Count (WBC), Absolute Neutrophil Count (ANC), Hemoglobin (Hgb), Platelets (PLT), Creatinine (Cr), and ALT, Total Bilirubin, Nasuea, Vomiting, and Weight changes. The Common Terminology Criteria Adverse Events (CTCAE) version 3.0 (Published August 9, 2006) was used for scoring these side effects, that included fever, fatigue, nausea, vomiting, diarrhea, sore mouth, loss of appetite, numbness and tingling.

\section{Inclusion Criteria}

Adult patients aged $>18$ years with an established diagnosis of cancer

Planned chemotherapy to be given during Ramadan

Signed informed consent form

Normal baseline CBC, RF, and LFT

\section{Exclusion Criteria}

Pregnancy

\section{Procedures}

\section{Recruitment}

Patients attending the Comprehensive Cancer Center at King Fahad Medical City their fasting status or their willingness to participate in the study were enrolled.

\section{Sample size:}

\section{STATISTICAL CONSIDERATION}

This pilot study focuses on the safety of fasting while receiving chemotherapy, using Common Terminology Criteria for Adverse Events (CTCAE) of the National Cancer Institute (NCI) as its primary outcome. Therefore, estimation of these safety parameters are the main goals rather than inference and results will be used to generate hypotheses for future studies. 


\section{Statistical Methods:}

The primary outcome variables of blood biomarkers difference values before and after fasting and nonfasting will be estimated with the corresponding 95\% confidence limits. These differences will be compared, on explorative basis, between fasting and non fasting using Normal distribution approximations and/or Wilcoxon non-parametric tests. Categorical values of symptoms before and after fasting (and non fasting) will be compared using Kappa McNamer tests and/or Kappa agreement statistic.

\section{RESULTS:}

Tables 1 and 2 displays the oncological description of the individual cases for the 11 patients along with their demographic characteristics. Eleven cancer patients receiving chemotherapy, 9 females and 2 males with a median age of 44 yrs (range 27-51 yrs), were recruited for this pilot study. Three suffered from breast cancer, two from Non- Hodgkin Lymphoma, and one from Acute Myeloid Leukemia, one each from Nasopharynx, Ovarian, and Colon cancer. (Table 1,2) Although it is small sample but it does represent most common malignancies around the world with considerable toxicity of the chemotherapeutic regimens use in these malignancies. While recruiting patients for this pilot study, we didn't deviate from the standard chemotherapy protocol used for management of these diseases. None of the patient discontinued treatment due to toxicity while fasting nor they required reduction in chemotherapy dosage.Patients were evaluated for symptoms and blood biomarkers pre and post chemotherapy while fasting and non fasting. (Table 3)We evaluated the pre and post non fasting and fasting blood biomarkers values along with their corresponding differences and in further contrast in these differences. Magnitude of the difference contrast between fasting and non-fasting blood biomarkers values was very minimal. Furthermore, all of the 95\% confidence intervals of these contrast contained the value zero which indicates lack of a significant difference. (Table 3) None of the patient while fasting developed grade 3 or 4 hematological, hepatic or renal toxicities.Fewer and less severe chemotherapy induced side effects were reported by all patients while fasting. In the comparison of the symptoms score based Common Terminology Criteria for Adverse Events of National Cancer Institute between fasting and non fasting. Nausea and vomiting are the most common side effects of most chemotherapy regimens, which lead to poor tolerability and compliance in patients with cancer. $12.5 \%$ of patient reported improvement in nausea while fasting. Fatigue is second most common symptom among patients on chemotherapy and one of the factor affecting functional class and quality of life. $50 \%$ of patients report subjective improvement in fatigue while fasting. Sore mouth another common symptom reported by patients while on chemotherapy which restricts patients from adequate nutrition and maintaining hydration. Patients reports better off while fasting around $62.5 \%$ felt better.(Table 4 )

Table 1. Demographic and clinical information of patients

\begin{tabular}{|l|c|c|c|l|}
\hline & Gender & Age & Primary Neoplasia & Stage at Diagnosis \\
\hline Case 1 & M & $50 \mathrm{yrs}$ & Nasopharynx & Stage III \\
\hline Case 2 & F & $38 \mathrm{yrs}$ & Breast & Stage IIB \\
\hline Case 3 & M & $33 \mathrm{yrs}$ & Colon & Stage IIB \\
\hline Case 4 & F & $27 \mathrm{yrs}$ & Hodgkin Lymphoma & Stage IIIB \\
\hline Case 5 & F & $51 \mathrm{yrs}$ & Follicular Lymphoma & Stage IIIA \\
\hline Case 6 & F & $28 \mathrm{yrs}$ & Acute Myeloid Leukemia & ----- \\
\hline Case 7 & F & $44 \mathrm{yrs}$ & Breast & Stage IIIB \\
\hline Case 8 & F & $42 \mathrm{yrs}$ & Breast & Stage IV \\
\hline Case 9 & F & $46 \mathrm{yrs}$ & Breast & Stage IV \\
\hline Case 10 & F & $51 \mathrm{yrs}$ & Ovary & Stage IIIC \\
\hline Case 11 & F & $51 \mathrm{yrs}$ & Diffuse Large B cell Lymphoma & Stage IV \\
\hline
\end{tabular}


Table 2. Additional data from patients, including chemotherapy protocol, co-morbidities and evaluation done at fasting and non fasting.

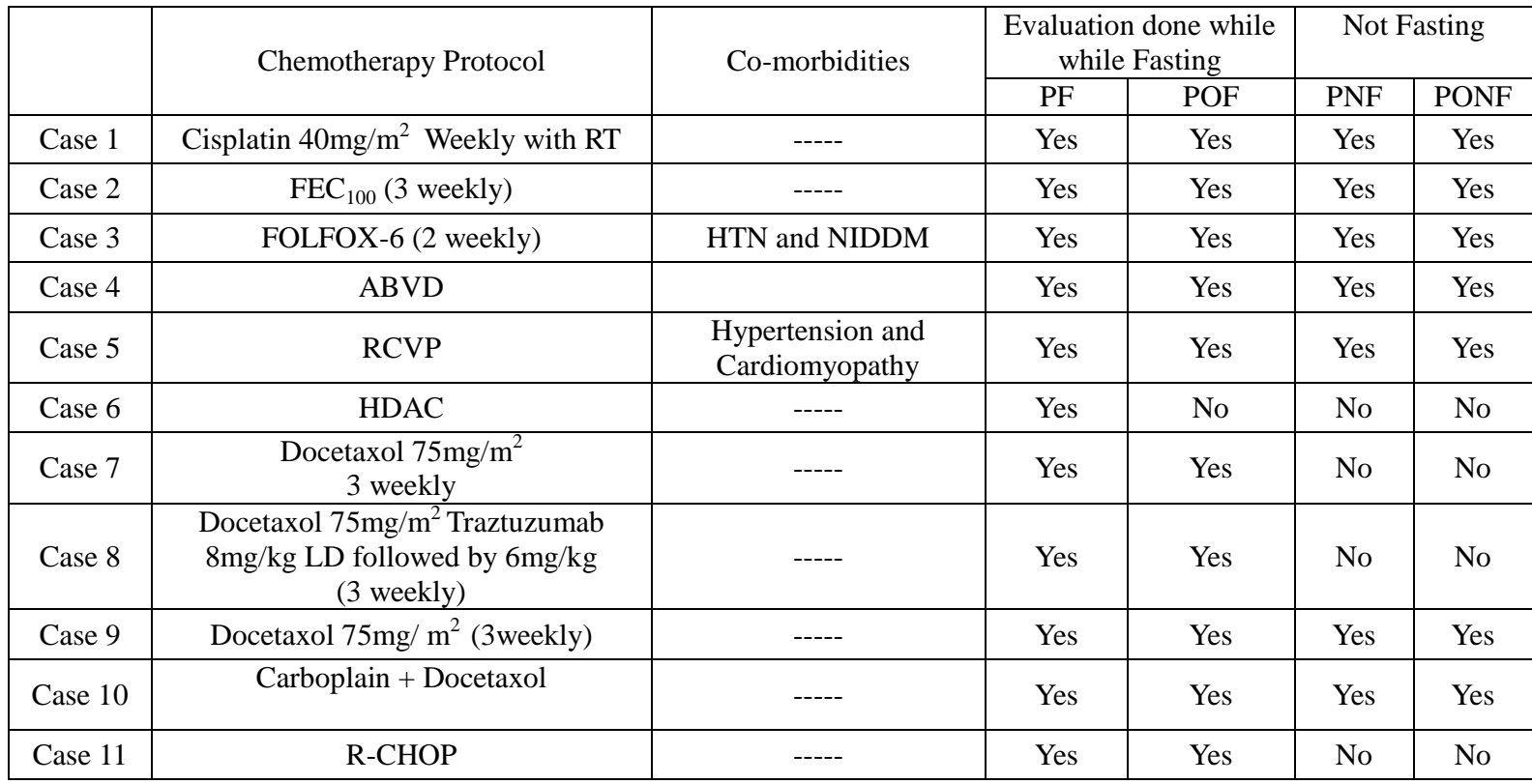

PF; Pre Fasting, POF; Post fasting; PNF; Pre non fasting, PONF; Post non fasting

RT; radiotherapy, $\mathrm{FEC}_{100}$; Flourouracil $500 \mathrm{mg} / \mathrm{m}^{2}+$ Epirubicin $100 \mathrm{mg} / \mathrm{m}^{2}+$ Cyclophosphamide $\quad 500 \mathrm{mg} / \mathrm{m}^{2}$, FOLFOX-6; Fluorouracil bolus followed by infusion + Oxaliplatin $85 \mathrm{mg} / \mathrm{m}^{2}$, ABVD; Doxorubicin $25 \mathrm{mg} / \mathrm{m}^{2}$ day 1 and $15+$ Bleomycin 10 units $/ \mathrm{m}^{2}$ day 1 and day $15+$ Vinblastin $6 \mathrm{mg} / \mathrm{m}^{2}$ day 1 and day $15+$ Dacarbazine $375 \mathrm{mg} / \mathrm{m} 2$ day 1 and day15.RCVP; Rituximab $375 \mathrm{mg} / \mathrm{m}^{2}+$ Cyclophosphamide $750 \mathrm{mg} / \mathrm{m}^{2}+$ Vincrstine $1.4 \mathrm{mg} / \mathrm{m}^{2}$ and Prednisolone $45 \mathrm{mg} / \mathrm{m}^{2}$ day 1 to day5. HDAC; High dose cytarabine $3 \mathrm{gm} / \mathrm{m}^{2}$. R-CHOP; Rituximab $375 \mathrm{mg} / \mathrm{m}^{2}+$ Cyclophosphamide $750 \mathrm{mg} / \mathrm{m}^{2}+$ Doxorubicin $50 \mathrm{mg} / \mathrm{m}^{2}+$ Vincristine $1.4 \mathrm{mg} / \mathrm{m}^{2}$ and Prednisolone $45 \mathrm{mg} / \mathrm{m}^{2}$ day $1-5$.

Table 3. Blood biomarkers difference values before and after fasting and non-fasting estimated with the corresponding $95 \%$ confidence limits.

Differences compared, on explorative basis, between fasting and non fasting using Normal distribution approximations and/or Wilcoxon non-parametric tests

\begin{tabular}{|c|c|c|c|c|c|c|c|c|c|c|}
\hline BB & $\begin{array}{l}\text { No.of } \\
\text { Cases }\end{array}$ & $\begin{array}{l}\text { Pre NF } \\
\text { (Mean) }\end{array}$ & $\begin{array}{c}\text { Post } \\
\text { NF } \\
\text { (Mean) }\end{array}$ & Diff. & $\begin{array}{l}\text { No. } \\
\text { of } \\
\text { Cases }\end{array}$ & $\begin{array}{l}\text { Pre F } \\
\text { (Mean) }\end{array}$ & $\begin{array}{l}\text { Post } F \\
\text { (Mean) }\end{array}$ & $\begin{array}{c}\text { Diff } \\
.\end{array}$ & $\mathrm{N}$ & $\begin{array}{c}\text { Diff, of Mean(NF-F) } \\
\text { Mean+/- } 95 \% \text { CI Std. } \\
\text { dev }\end{array}$ \\
\hline WBC & 7 & 4.85 & 4.67 & 0.18 & 11 & 5.65 & 3.76 & 1.89 & 7 & $-0.30 \quad(-3.87,3.25)$ \\
\hline ANC & 7 & 2.16 & 2.15 & 0.01 & 11 & 2.95 & 1.80 & 1.15 & 7 & $-0.14(-2.89,2.57)$ \\
\hline $\mathrm{Hb}$ & 7 & 11.60 & 11.30 & 0.30 & 11 & 12.10 & 11.60 & 0.50 & 7 & $-0.09 \quad(-0.75,0.55)$ \\
\hline Plt & 7 & 290.0 & 313.5 & -23.5 & 11 & 332.1 & 254.5 & 77.6 & 7 & $-72.71(-143.5,-1.95)$ \\
\hline $\mathrm{Cr}$ & 7 & 62 & 55 & 7 & 11 & 58 & 59 & -1.0 & 7 & $5.28(-2.54,13.10)$ \\
\hline T.Bili & 7 & 4.9 & 5.5 & 0.6 & 11 & 5.0 & 6.5 & -1.5 & 7 & $0.08 \quad(-3.75,3.93)$ \\
\hline $\begin{array}{c}\text { SGP } \\
\mathrm{T}\end{array}$ & 7 & 40.9 & 46.7 & -5.8 & 11 & 39.3 & 44.2 & -4.9 & 7 & $-3.8(-18.9,-11.2)$ \\
\hline Wt. & 7 & 78.7 & 78.6 & 0.1 & 11 & 73.7 & 74.4 & -0.7 & 7 & $1.04(-0.77,2.85)$ \\
\hline
\end{tabular}


Table 4. Self reported side effects after chemotherapy with or without fasting.

Categorical values of symptoms before and after fasting (and non fasting) compared using Kappa McNamer tests and/or Kappa agreement statistic.

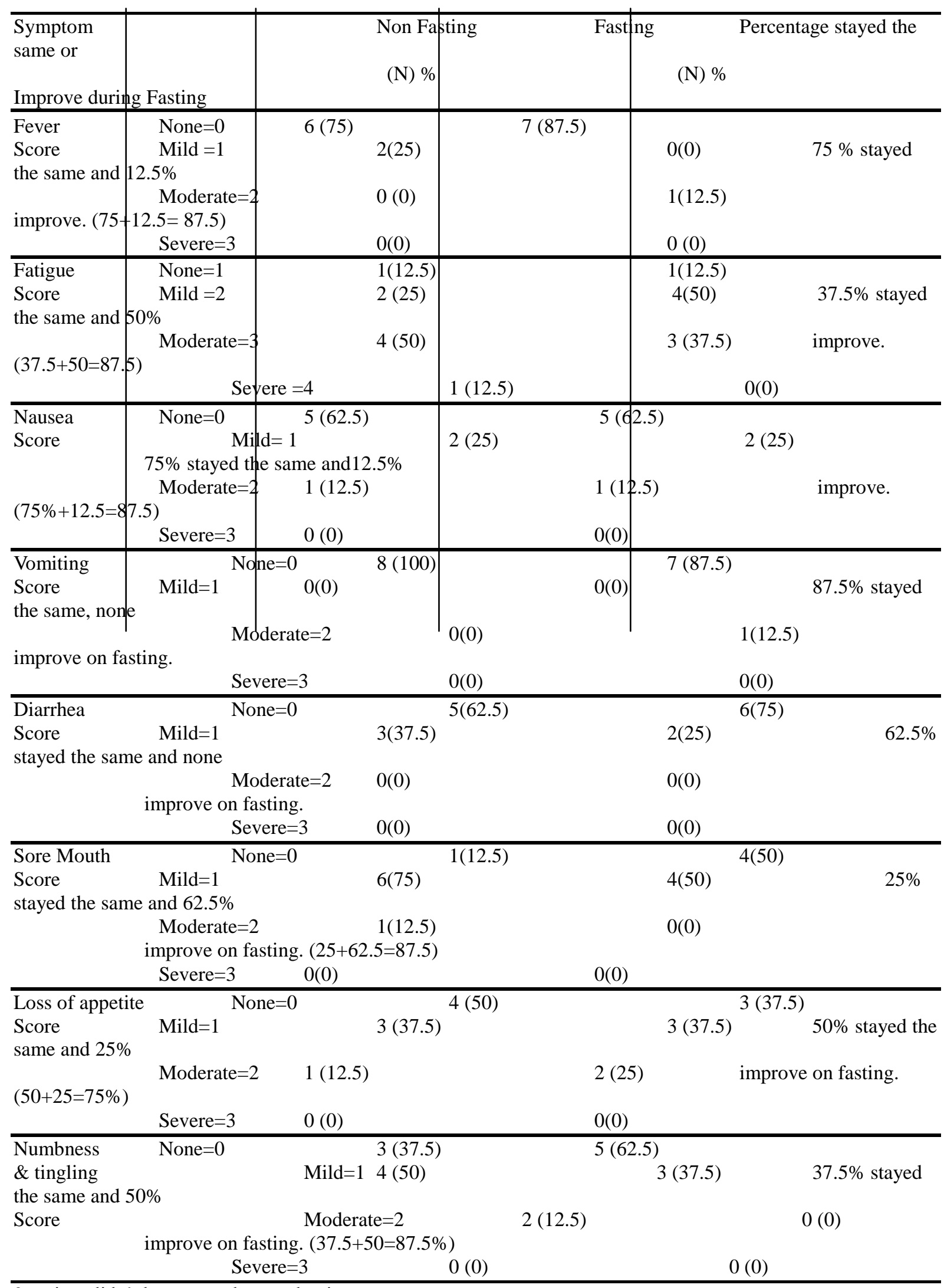

3 patient didn't have complete evaluation 


\section{DISCUSSION:}

Chemotherapy can improve survival in patient diagnosed with malignancies. However chemotherapy induced toxicity to normal cells limit chemotherapy dose, which may decrease efficacy. Hence, reduction of undesired side effects by selective protection of normal cells without compromising the killing of cancer cells by chemotherapy represents a promising strategy to enhance cancer treatment. Calorie restriction (CR) is an effective and reproducible intervention for decreasing incidence, burden and cancer multiplicity in various species (rats and mice) $)^{3-6}$ Caloric restriction (CR) has shown to be well tolerated in humans as wells as in children. ${ }^{9-12}$ Furthermore CR has shown to decrease oxidative stress to normal cells, enhance cancer killing by chemotherapeutic agent while protecting normal cells. ${ }^{7-8,10-12}$ Dietary recommendation during cancer treatment is based on prevention and reversal of nutrient deficiencies and side effects related to it. Cancer patients who are nutritionally compromised, physicians may consider a fasting based strategy to be devastating. Hence, the American Cancer Society recommends that cancer patients receiving chemotherapy should increase calorie and protein intake. ${ }^{13}$ Nevertheless, studies have shown that $20-40 \%$ reduction in calorie intake protects the host against toxins and retards the growth of tumors. ${ }^{14}$ A case series report of patients with heterogeneous cancers tolerated fasting repeatedly in multiple cycles for up to $180 \mathrm{hrs}$ with comparable side effects without affecting quality of life. ${ }^{12}$ In our exploratory pilot study, several patients diagnosed with wide variety of cancer, elected to undertake Muslim fasting from dawn to sunset while receiving chemotherapy, which is an intermittent rhythmic calorie restriction that is unique and difficult to find comparable group with the same practice which include deprivation to even water.

In this small group, minor complaints of nausea, vomiting, diarrhea, loss of appetite and sore mouth reported at a level that did not interfere with daily activities. We didn't report serious complications among our patients in fasting group. We also compare changes in the blood biomarkers while fasting and non fasting. We did not find alarming spikes / changes in blood biomarkers or deterioration of symptoms during fasting compare to non fasting. Considering small group with significant limitations no format test of hypotheses is sought. We are aware of the fact that lack of statistical differences can; largely be due to smaller sample size.

In conclusion, in this small and heterogonous group of patients, fasting was well tolerated and comparable even reduction in side effects compare to non fasting. Although bias could affect the findings in the study but findings of the study are in accordance with the results obtained from animal studies. This series of patients can provide early insight into the feasibility of fasting while receiving chemotherapy and will certainly open room for future studies.

REFERENCES:

[1] WCRF/AICR; Food, nutrition and the prevention of cancer: a global perspective:. World Cancer Research Fund/ American Institute for Cancer Research 1997.

[2] Dirx M. , Zeegers M. , Dagnelie P. , van den Bogaard T. and van den Brandt P. " energy restriction and the risk of spontaneous mammary tumors in mice: a meta-analysis" Int. J. Cancer 2003; 106, 766-770

[3] Klurfeld, D. M., Welch, D. B., Davis, M. J. \& Kritchevsky, D. "Determination of degree of energy restriction necessary to reduce DMBA-induced mammary tumorigenesis in rats during the promotion phase.” J. Nutr. 1989; 119: 286-291

[4] Hursting, S. D., Perkins, S. N., and Phang, J. M. "Caloric restriction delays spontaneous tumorigenesis in p53 knockout transgenic mice.” Proc. Natl. Acad. Sci. USA, 1994; 91: 7036-7040.

[5] Ross, M. H. \& Bras, G. "Lasting influence of early caloric restriction on prevalence of neoplasms in the rat." J. Natl. Cancer Inst. 1971; 47: 1095-1113

[6] Weindruch, R. \& Walford, R. L. "Dietary restriction in mice beginning at 1 year of age: effect on life span and spontaneous cancer incidence." Science 1982; 215: 1415-1418.

[7] Raffaghello L., Lee C., Safdie F.M., Wei M., Madia F., Bianchi G., and Longo V.D. "Starvation-dependent differential stress resistance protects normal but not cancer cells against high-dose chemotherapy". Proc. Natl. Acad. Sci. USA, 2008; 105: 82158220

[8] Lee C, Safdie FM, Raffaghello L, Wei M, Madia F, Parrella E et al. Reduced levels of IGF-I mediate differential protection of normal and cancer cells in response to fasting and improve chemotherapeutic index. Cancer Res 70,2010: 1564-1572. Cited in $\mathrm{C}$ Lee and VD Longo Fasting vs dietary restriction in cellular protection and cancer treatment: from model organisms to patients. Oncogene (2011) 30, 3305-3316

[9] James B. Johnson, Warren Summer, Roy G. Cutler, Bronwen Martin,Dong-Hoon Hyun, Vishwa D. Dixit,Michelle Pearson,Matthew Nassar,Stuart Maudsley, Olga Carlson, Sujit John, Donald R. Laub, and Mark P. Mattson. Alternate Day Calorie Restriction Improves Clinical Findings and Reduces Markers of Oxidative Stress and Inflammation in Overweight Adults with Moderate Asthma. Free Radic Biol Med. 2007 March 1; 42(5): 665-674.

[10] Katz LE, DeLeón DD, Zhao H, Jawad AF. Free and total insulin-like growth factor (IGF)-I levels decline during fasting: relationships with insulin and IGF-binding protein-1. J Clin Endocrinol Metab. 2002 Jun;87(6):2978-83.

[11] Luigi Fontana,Timothy E. Meyer, Samuel Klein, and John O. Holloszy

[12] Long-term calorie restriction is highly effective in reducing the risk for atherosclerosis in humans. Proc Natl Acad Sci U S A. 2004 April 27; 101(17): 6659-6663.

[13] Fernando M. Safdie, Tanya Dorff, David Quinn, Luigi Fontana, Min Wei1, Changhan Lee, Pinchas Cohen, and Valter D. Longo .Fasting and cancer treatment in humans: A case series report. AGING. Dec 2009; vol.1No12.

[14] 13 Doyle C, Kushi LH, Byers T, Courneya KS, Demark-Wahnefried W, Grant B et al. (2006). Nutrition and physical activity during and after cancer treatment: an American Cancer Society guide for informed choices. CA Cancer J Clin 56: 323-353.

[15] Lee, C., Longo, V.D., 2011. Fasting vs dietary restriction in cellular protection and cancer treatment: from model organisms to patients. Oncogene $30,3305-3316$. 